\title{
Characterization of Apomictic Potential in Guayule (Parthenium argentatum) In Vivo and In Vitro
}

\author{
Roy N. Keys ${ }^{1}$ and Dennis T. Ray ${ }^{2}$ \\ Department of Plant Sciences, The University of Arizona, Tucson, AZ 85721-0036 \\ David A. Dierig \\ U.S. Department of Agriculture, Agricultural Research Service, U.S. Water Conservation Laboratory, \\ 4331 E. Broadway Road., Phoenix, AZ 85040
}

AdDitional InDEX WORDS. apomixis, mitotic diplospory, Asteraceae, auxin, ovule culture

\begin{abstract}
Aвstract. Guayule (Parthenium argentatum Gray), a latex-producing perennial desert shrub and potential industrial crop for semiarid regions, exhibits reproductive modes ranging from sexual, self-sterile diploids to predominantly apomictic, self-compatible polyploids. The objectives of this study were to develop and evaluate a rapid, simple technique for characterizing apomictic potential (percentage of ovules that produce apomictic embryos) in guayule breeding lines. Initial in vivo experiments were based on an auxin test that permitted quantification of apomictic frequency in grasses. In our trials, floral application of NAA or IBA resulted in embryo production similar to that of open-pollinated controls, but 2,4-D inhibited embryo production. Breeding lines could be separated based on embryo production using an in vivo auxin test; however, accuracy of the results was questionable because 1) pollen release and insect activity within isolation bags prevented distinguishing between sexual and apomictic embryos, and 2) high temperatures and large humidity fluctuations could have affected results. Thus, in vitro flower culture was investigated using liquid medium, because it would provide better control of these factors. Flowers developed normally in vitro, except that pollen was not released from the anthers; therefore, any embryos produced in vitro were considered to be apomictic. Embryo production was similar on both Nitsch and Nitsch and Woody Plant Media. Addition of growth regulators inhibited embryo production. Embryo production was tested on Nitsch and Nitsch medium without growth regulators for seven breeding lines. Based on statistical analyses, four classes of apomictic potential were identified, ranging from none (sexual) to high. Chemical names used: 2,4-dichlorophenoxy acetic acid (2,4-D); indole-3-butyric acid (IBA); $\alpha$-naphthaleneacetic acid (NAA).
\end{abstract}

Guayule (Parthenium argentatum) is a latex-producing, perennial desert shrub native to southwest Texas and north-central Mexico. With the advantages of having relatively low water requirements and producing nonallergenic latex, it represents an economically viable industrial crop for semiarid regions (Thompson and Ray, 1988). Diploid guayule plants reproduce predominantly sexually and possess a sporophytic self-incompatibility system (Gerstel, 1950; Gerstel and Riner, 1950; Powers and Rollins, 1945). Polyploid plants are self-compatible and can reproduce by facultative or obligate pseudogamous apomixis, predominantly through mitotic diplospory (Esau, 1946; Powers and Rollins, 1945). The frequency of apomictic reproduction can vary among plants, and apparently even among flowers on a single plant (Esau, 1946). However, the factors that control the mode of reproduction are unknown.

Flowers of guayule (Asteraceae) are arranged in heads, with a variable number of heads borne on a panicle (Artschwager, 1943). The internal disk flowers are staminate, and there are usually five peripheral pistillate ray flowers. There is a slight tendency toward protogyny since flowers mature centripetally. However, there are two disk flowers adnate to each ray flower that mature at about the same time as the ray flower. This floral structure and pattern of development, together with the small floral size, make emasculation difficult.

Guayule breeding programs would be facilitated by the ability to estimate the frequency of apomixis (the percentage of ovules that produce apomictic embryos), or apomictic potential (hereafter referred to as AP), in breeding lines. Traditional methods of plant microtechnique used by Esau (1946), while providing reliable characterization of reproductive mode, are not practical on a large

Received for publication 31 July 2001. Accepted for publication 12 Feb. 2002. ${ }^{1}$ Current address: 746 Berry Patch Way, Southington, CT 06489.

${ }^{2}$ Corresponding author. scale. Therefore, a relatively rapid and easy technique to determine $\mathrm{AP}$ in guayule would be beneficial and the objective of this research was to develop and test such a technique.

A simple technique has been used to distinguish apomictic and sexual genotypes in the Poaceae (Matzk, 1991; Mazzucato et al., 1996). This technique is based on the observation that auxins stimulate development of apomictic embryos in plants that are genetically disposed toward apomixis, but not in those that are truly sexual. In the Poaceae, apomictic embryos developed to apparent maturity in the absence of endosperm after auxin treatment, permitting easy visual evaluation of test results (Matzk, 1991; Mazzucato et al., 1996).

We applied the above auxin test (Matzk, 1991; Mazzucato et al., 1996) in field trials to determine the AP of guayule breeding lines. Differences in percentage embryo production among lines could be detected in vivo. The accuracy of these results was, however, questionable because 1) pollen release and insect activity within the isolation bags prevented clear distinction between apomictic and sexual embryos, and 2) high temperatures and large fluctuations in humidity could have had adverse effects. Thus to address these problems, an in vitro flower culture technique for determining AP in guayule was developed. This technique was used to characterize the AP of seven polyploid breeding lines and a bulked diploid (sexual) control.

\section{Materials and Methods}

\section{In vivo trials}

Plant Materials. Three in vivo experiments were conducted. In vivo experiments were conducted on 1-year-old plants grown at the University of Arizona Marana Agricultural Center, $50 \mathrm{~km}$ north of Tucson. Seven open-pollinated lines were tested (G7-11, G7-14, G7-15, N7-11, N9-5, P2-BK, and P10-13). Variability within lines 
ranged from uniform to highly variable. Four of these lines have been released and registered as improved germplasm (Ray et al., 1999). The plants were all assumed to be tetraploids because Cho and Ray (unpublished data) found that other open-pollinated progeny derived from the same parental stocks as the plants used in this study were predominantly tetraploid $(2 n=4 x=72)$, with a small proportion either triploid or polyhaploid.

Procedures. In vivo experiments were conducted from July to October 1996. For each experiment, all seven breeding lines were tested. Panicles that contained a few heads on which the ray flowers were at or beyond anthesis were selected for treatment. The most mature and most immature flower heads were removed from each panicle to leave three flower heads of approximately the same developmental stage. This also ensured that none of the stigmas had been exposed to pollen. Flower heads were treated by dipping the entire panicle into a treatment solution for $5 \mathrm{~s}$. The panicle was then covered with an isolation bag to prevent cross-pollination.

In VIVO EXPT. 1. The basic treatment, based on that of Mazzucato et al. (1996), was $0.36 \mathrm{~mm} \mathrm{2,4-D}$ in $0.1 \%$ Tween 20 (polyoxyethylenesorbitan). Two controls were used, $0.1 \%(\mathrm{v} / \mathrm{v})$ Tween 20 and open-pollination, which provided minimum and maximum estimates of embryo production. The experiment was replicated four times over time.

IN VIVO EXPT. 2. There were three treatments of $0.18,0.36$, or 0.54 mM 2,4-D in 30\% ethanol. Ethanol was used in place of Tween 20 in this trial because ancillary tests indicated it reduced pollen release without significant adverse affects on floral development (Keys and Ray, unpublished data). Two controls were used: $30 \%$ (v/v) ethanol and open-pollination. The experiment was replicated three times over time.

IN VIVO EXPT. 3. Treatments were $0.1 \mathrm{~mm}$ of either IBA, NAA, or 2,4-D in $30 \%(\mathrm{v} / \mathrm{v})$ ethanol. Two controls were used: $30 \%(\mathrm{v} / \mathrm{v})$ ethanol and open-pollination. The experiment was replicated twice over time.

All experiments used a completely randomized design. Plants were randomly selected for each experiment, so that separate experiments were not necessarily conducted on the same plants. The flower heads were collected at $14 \mathrm{~d}$ for evaluation of the percentage of flowers containing embryos.

\section{In vitro trials}

Plant Materials. Three in vitro tests were conducted. For in vitro investigations, randomly selected plants from the same seven lines described above were transplanted from the field into pots in November 1996. The shoots of the selected plants were clipped to $15 \mathrm{~cm}$ of the soil surface, and roots were harvested down to $30 \mathrm{~cm}$ of the soil surface. Six known diploid plants were used as a bulked sexual control in these studies. The transplants had regrown and were flowering (ready for treatments) $\approx 1$ month after being transferred to the greenhouse. The plants were grown in a medium of 1 sand : 1 perlite : 1 peat (by volume) in 12 - to 20 -L pots under greenhouse conditions (minimum/maximum temperatures of 23/32 ${ }^{\circ} \mathrm{C}$ ). Irrigation was provided by an overhead mist system. Grolux (40 W, Sylvania Electronic Products, Inc., Salem, Mass.) fluorescent lamps were placed $30 \mathrm{~cm}$ above the plants to provide a 14-h photoperiod during winter months to induce flowering. Osmocote slow release (14N-4.2P-11.6K; Scotts-Sierra Hort. Products Co., Marysville, Ohio) was applied at $12 \mathrm{~g} /$ container about every 3 months.

Culture Procedures. Panicles were collected on the day of culture initiation. Individual flower heads were selected based on the stage of development. The best stage was considered to be when the ray flowers had begun to open and their stigmas were at least partially visible, but before the stigma began to spread open into the two surfaces it normally presents at maturity. As an added safeguard against pollination, each flower head was examined under a dissecting microscope for the presence of pollen on the stigmas. The entire flower head was then sterilized by placing it in $0.79 \%(\mathrm{v} / \mathrm{v})$ sodium hypochlorite (15\% commercial bleach) and $0.1 \%$ (v/v) Tween-20 for $3 \mathrm{~min}$, with agitation. The heads were rinsed in sterile, deionized, distilled water and placed in the culture medium. Each flower head was cultured in $2.5 \mathrm{~mL}$ of autoclaved liquid medium in a $2.5 \times 6.5$ $\mathrm{cm}$ glass vial sealed with a plastic cap. The cultures were placed on a shaker, at $80 \mathrm{rpm}$, on a laboratory bench, and grown at room temperature $\left(22^{\circ} \mathrm{C} \pm 2{ }^{\circ} \mathrm{C}\right)$. The cultures were irradiated continuously by fluorescent light with an average PPF at culture level of 5 $\mu \mathrm{mol} \cdot \mathrm{m}^{-2} \cdot \mathrm{s}^{-1}$ (quantum meter; Apogee Instruments, Logan, Utah).

IN VITRO EXPT. 1. This was a preliminary test on the effect of growth regulators on embryo production. The medium consisted of Nitsch and Nitsch (NN) (1969) major and minor salts, vitamins, and $180 \mathrm{~mm}$ sucrose, $\mathrm{pH}$ 5.8. It was used either alone or supplemented with NAA, NAA and gibberellic acid $\left(\mathrm{GA}_{3}\right)$, or NAA and kinetin all at $1.0 \mu \mathrm{M}$. This test was not replicated, and used one flower head per treatment from each of five lines.

IN VITRo EXPT. 2. Two media were tested: NN and woody plant medium (WP) (Lloyd and McCown, 1980). Both media contained $180 \mathrm{~mm}$ sucrose, no growth regulators, and $\mathrm{pH}$ was adjusted to 5.8. This experiment was replicated from two to seven times using six of the polyploid lines and five diploid plants, for a total of 31 flower heads per treatment.

IN VITRO EXPT. 3. The apomictic potential of the seven breeding lines was characterized using NN medium without growth regulators. Four to twelve plants were sampled within lines, and the experiment was replicated two to five times per plant, depending on the availability of flowers.

All three experiments used a completely randomized design. The flower heads were evaluated after $14 \mathrm{~d}$ in culture for percentage embryo production.

PostTREatment EVAluation OF POLLEN PRESENCE AND EMBRYo PRODUCTION. For both in vivo and in vitro trials, ray flowers were removed from the flower heads and examined microscopically for the presence of pollen. If pollen was observed on the stigma, it was scored as present. For flowers grown in vitro, floral development was scored as $0=$ no obvious development; $1=$ some development of either the ray or disk flowers; and $2=$ apparently complete development of both ray and disk flowers (even if pollen had not been released). The single ovule was excised from each flower and placed in water on a microscope slide. After covering with a cover slip, the ovule was carefully squashed and the contents examined using phase contrast microscopy. Proembryos and embryos could be detected in this way, and were recorded when present.

STATISTICAL ANALYSES. The percentage of flowers producing embryos was transformed using the arcsine of the square root to permit analysis of variance. Data were analyzed using General Linear Models Procedure of SAS (SAS Institute Inc., 1988). Means were separated using Duncan's multiple range test, orthogonal contrasts were performed for each breeding line against the bulked data for the diploid controls, and a chi-square test for heterogeneity among lines was conducted.

\section{Results}

In reading this section, keep in mind that the results of one experiment were used as a basis for design of the succeeding 
Table 1. Effect of Tween 20 with and without 2,4-D on embryo production in flowers of field-grown guayule compared to an open-pollinated control (combined means for seven breeding lines) (in vivo Expt. 1).

\begin{tabular}{|c|c|c|}
\hline Treatment $^{z}$ & $\begin{array}{c}\text { Flower } \\
\text { heads (no.) }\end{array}$ & $\begin{array}{c}\text { Flowers with } \\
\text { embryos } \\
(\% \pm \mathrm{SE})\end{array}$ \\
\hline Open-pollinated & 30 & $33 \mathrm{a}^{\mathrm{y}}( \pm 4)$ \\
\hline $0.36 \mathrm{~mm} 2,4-\mathrm{D}$ in $0.1 \%$ Tween $20^{\mathrm{x}}$ & 23 & $12 b( \pm 3)$ \\
\hline $0.1 \%$ Tween $20^{z}$ & 20 & $12 \mathrm{~b}( \pm 3)$ \\
\hline
\end{tabular}

ZTreatments applied as a 5-s dip.

yean separation within column by Duncan's multiple range test, $P \leq 0.05$.

xPolyoxyethylenesorbitan.

Table 2. Effects of ethanol, with and without 2,4-D at three concentrations, on embryo production in flowers of field-grown guayule in comparison to an open-pollinated control (combined means for seven breeding lines) (in vivo Expt. 2).

\begin{tabular}{lcc}
\hline \hline & $\begin{array}{c}\text { Flower } \\
\text { heads (no.) }\end{array}$ & $\begin{array}{c}\text { Flowers with } \\
\text { embryos } \\
(\% \pm \mathrm{SE})\end{array}$ \\
\hline Oreatment ${ }^{\mathrm{z}}$ & 21 & $20 \mathrm{a}^{\mathrm{y}}( \pm 3)$ \\
$30 \%$ ethanol & 21 & $15 \mathrm{ab}( \pm 3)$ \\
$0.51 \mathrm{mM}$ 2,4-D in 30\% ethanol & 16 & $6 \mathrm{bc}( \pm 3)$ \\
$0.36 \mathrm{mM}$ 2,4-D in 30\% ethanol & 18 & $5 \mathrm{c}( \pm 2)$ \\
$0.18 \mathrm{mM}$ 2,4-D in 30\% ethanol & 5 & $3 \mathrm{c}( \pm 3)$ \\
\hline
\end{tabular}

zTreatments applied as a 5-s dip.

${ }^{\text {y} M e a n ~ s e p a r a t i o n ~ w i t h i n ~ c o l u m n ~ b y ~ D u n c a n ' s ~ m u l t i p l e ~ r a n g e ~ t e s t, ~} P \leq 0.05$.

Table 3. Effects of ethanol, with and without auxin, on embryo production in flowers of field-grown guayule in comparison to an open-pollinated control (combined means for seven breeding lines) (in vivo Expt. 3).

\begin{tabular}{|c|c|c|}
\hline Treatment $^{2}$ & $\begin{array}{c}\text { Flower } \\
\text { heads (no.) }\end{array}$ & $\begin{array}{c}\text { Flowers with } \\
\text { embryos } \\
(\% \pm \mathrm{SE})\end{array}$ \\
\hline$\overline{\text { Open-pollinated }}$ & 18 & $23 \mathrm{a}^{\mathrm{y}}( \pm 5)$ \\
\hline IBA in $30 \%$ ethanol & 27 & 28 a $( \pm 6)$ \\
\hline NAA in $30 \%$ ethanol & 21 & 25 a $( \pm 6)$ \\
\hline $30 \%$ ethanol & 24 & $16 \mathrm{ab}( \pm 5)$ \\
\hline $2,4-\mathrm{D}$ in $30 \%$ ethanol & 27 & $6 \mathrm{~b}( \pm 2)$ \\
\hline
\end{tabular}

zTreatments applied as a 5-s dip, with all auxins at a concentration of 0.1 $\mathrm{mM}$.

${ }^{\text {y} M e a n ~ s e p a r a t i o n ~ w i t h i n ~ c o l u m n ~ b y ~ D u n c a n ' s ~ m u l t i p l e ~ r a n g e ~ t e s t, ~} P \leq 0.05$.

Table 4. Embryo production, averaged across treatments, in flowers of seven field-grown breeding lines of guayule (in vivo Expt. 3).

\begin{tabular}{lcc}
\hline \hline Line & $\begin{array}{c}\text { Flower } \\
\text { heads (no.) }\end{array}$ & $\begin{array}{c}\text { Flowers with } \\
\text { embryos } \\
(\% \pm \text { SE) }\end{array}$ \\
\hline G7-11 & 12 & $33 \mathrm{a}^{\mathrm{y}}( \pm 7)$ \\
N7-11 & 12 & $32 \mathrm{a}( \pm 10)$ \\
P10-13 & 21 & $26 \mathrm{ab}( \pm 6)$ \\
P2-BK & 24 & $16 \mathrm{abc}( \pm 4)$ \\
G7-15 & 12 & $13 \mathrm{bc}( \pm 8)$ \\
N9-5 & 18 & $11 \mathrm{bc}( \pm 4)$ \\
G7-14 & 18 & $10 \mathrm{c}( \pm 4)$ \\
\hline
\end{tabular}

$\overline{\mathrm{z}}$ Values are means per line across five treatments.

${ }^{\mathrm{y}}$ Mean separation within column by Duncan's multiple range test, $P \leq 0.05$. experiment. In this way, evolution of the final technique can be seen in the progression from in vivo to in vitro experiments.

IN VIVO EXPT. 1. There were significant differences among treatment means with most embryos being produced in the openpollinated control (Table 1). Regardless of treatment with 2,4-D, the Tween 20 dip reduced embryo production. Differences among lines and treatment by line interactions for embryo production were nonsignificant. Pollen was released in $50 \%$ of the Tween 20 treated and $35 \%$ of the 2,4-D treated inflorescences. Insect activity, mainly thrips (Frankliniella occidentalis Pergande), within isolation bags apparently helped spread the pollen. These factors prevented clear distinction between apomictic and sexual embryos.

IN VIVO EXPT. 2. All three concentrations of 2,4-D reduced embryo production compared to the open-pollinated control (Table 2 ). Differences among lines and treatment by line interaction for embryo production were nonsignificant. When ethanol was substituted for Tween 20 as a carrier for 2,4-D, there was no significant difference between the open-pollinated control and the ethanol control. Ethanol and ethanol in combination with 2,4-D inhibited pollen release, but pollen release still occurred in $38 \%$ and $20 \%$ of the flower heads, respectively. Epidermal floral tissues responded to the auxin, as evidenced by swelling. This response occurred in conjunction with deterioration of the ovular tissues, so much that it was often difficult to identify embryos with certainty in the $0.54 \mathrm{~mm}$ treatment.

IN VIVo EXPT. 3. Embryo production in the IBA and NAA treatments was similar to that of both the open-pollinated and ethanol controls (Table 3). It was, however, significantly lower in the 2,4-D treatment than for the other two auxins. Differences among lines for embryo production were detected (Table 4), and there was a significant treatment by line interaction. Pollen release was low $(17 \%)$ in the 2,4-D treatment, but the ovular tissues were severely deteriorated. Pollen release occurred in $100 \%$ of the other auxin treatments, preventing clear distinction between apomictic and sexual embryos.

IN VITRO EXPT. 1. No significant differences in embryo production occurred among treatments, probably because of the small sample size (Table 5). However, addition of NAA to the medium reduced embryo production by more than half. Combinations of either $\mathrm{GA}_{3}$ or kinetin with NAA resulted in a further reduction in embryo formation. Flowers developed normally in vitro, even exhibiting the dark brown pigmentation of epidermal layers that occurs in vivo as the flowers mature. Although pollen developed in the anthers, microscopic examination revealed a complete lack of pollen release in the vials. Therefore, all embryos were considered to be apomictic.

IN VITRo EXPT. 2. There was no significant effect of medium on embryo production. On WP medium, $23 \%(\mathrm{SE}=0.06)$ of the flowers produced embryos, while on $\mathrm{NN}$ medium $22 \%(\mathrm{SE}=0.05)$ produced embryos. Microscopic examination revealed a complete lack of pollen release in the vials.

IN VITRO EXPT. 3. There were significant differences among lines for embryo production (Table 6). Orthogonal contrasts of presumed polyploid lines against the bulked diploid controls, in combination with mean separations based on Duncan's multiple range test, permitted assignment of the lines to four AP classes, as follows: High $=$ mean embryo production significantly different from that of the diploid control, according to both Duncan's multiple range test and orthogonal contrast $(P \leq 0.05)$; Medium $=$ mean embryo production not significantly different that of either the High group or the diploid control, according to Duncan's multiple range test; Low $=$ mean embryo production significantly different from that 
Table 5. Effects of NAA, GA 3 , and kinetin, alone or in combination, on embryo production in flowers of guayule grown in vitro, in comparison to a control without growth regulators (in vitro Expt. 1).

\begin{tabular}{lcc}
\hline \hline Treatment & $\begin{array}{c}\text { Flower } \\
\text { heads }(\text { no. })\end{array}$ & $\begin{array}{c}\text { Flowers with } \\
\text { embryos } \\
(\% \pm \mathrm{SE})\end{array}$ \\
\hline No growth regulator & 5 & $32 \mathrm{a}^{\mathrm{z}( \pm 16)}$ \\
$1.0 \mu \mathrm{M}$ NAA & 5 & $12 \mathrm{a}( \pm 12)$ \\
$1.0 \mu \mathrm{M}$ each NAA and GA & 5 & $4 \mathrm{a}( \pm 4)$ \\
$1.0 \mu \mathrm{M}$ each NAA and kinetin & 5 & $4 \mathrm{a}( \pm 4)$ \\
\hline
\end{tabular}

of the High group, but not from that of the diploid control, according to Duncan's multiple range test; and None (sexual): no embryo production. Microscopic examination revealed a complete lack of pollen release in the vials; therefore, all embryos were considered to be apomictic.

Although not statistically significant, there was important withinline variation in expression of apomixis. For example, in line N9-5, which was in the High AP class, one plant never produced embryos in vitro. In contrast, line P10-13 was in the Low AP class. While most of the plants in this line did not produce embryos in vitro, a few plants did.

Thirty-seven percent of the flower heads placed in culture did not develop. A chi-square test for heterogeneity among the lines was conducted and was nonsignificant $\left(\chi^{2}=11.1, P=0.08\right)$, showing that this phenomenon was not related to AP.

\section{Discussion}

IN VIVO EXPERIMENTS. Embryo production was highest using either IBA or NAA, and lowest using 2,4-D. This is in contrast to responses reported in six genera of the Poaceae, in which the frequency of parthenogenesis was greater with 2,4-D than with either IBA or NAA (Matzk, 1991). Even at low concentrations, the effects of 2,4-D, on floral development were to 1) cause swelling of epidermal tissues and trichomes at the expense of ovular development, and 2) inhibit anthesis. IBA and NAA did not have these effects, and they actually overcame the slight inhibition of anthesis caused by the ethanol carrier.
The in vivo auxin test presented some technical problems in guayule. First, pollen release and insect activity that occurred in the isolation bags could have resulted in sexual embryo production, because polyploid guayule can be self-fertile (Powers and Rollins, 1945). The importance of this factor on in vivo results increases when one considers that variation among lines in self-fertility could exist. Variation in self-fertility and potential self-pollination could have contributed to the treatment by line interaction detected in this trial. In order to overcome this problem, plants would have to be characterized for self-fertility in conjunction with the in vivo auxin test. Second, adverse environmental conditions, caused by extremely high temperatures and large fluctuations in humidity that are typical of the summer rainy season in Arizona, could have affected results in vivo. Mamood et al. (1990) found seasonal fluctuation in seed production in guayule that could be the result of these same factors.

IN VITRO EXPERIMENTS. In light of the technical problems encountered with the in vivo trials, as discussed above, we considered that in vitro flower culture would permit control of temperature and humidity, as well as light. Insects would also be excluded. An additional, unexpected benefit of in vitro culture was that, even though pistils and stamens appeared to develop normally, pollen was never released in the vials. Thus, self-pollination was eliminated as a factor.

The inhibitory effects of auxin application on embryo production in guayule flowers grown in vitro were evident with inclusion of NAA in the culture medium. The level of embryo production in the absence of growth regulators was similar to that occurring in nontreated (open-pollinated) flowers in vivo (Tables 1-3). Inclusion of NAA alone reduced embryo production, and in combination with other growth regulators resulted in a further reduction in embryo production. Apparently, endogenous levels of growth regulators in the floral tissues are sufficient for embryo production. External application of growth regulators may cause either an inhibitory threshold to be exceeded, or a disruption of the balance of endogenous growth regulators so that apomictic development is disturbed. Failure of $\mathrm{GA}_{3}$ and cytokinin to stimulate embryo production was also reported in the Poaceae (Matzk, 1991).

The composition of the basal medium (NN vs. WP) did not affect embryo production in vitro. Espinasse et al. (1991), in a test of nutrient media that also included $\mathrm{NN}$ medium, reported a similar

Table 6. Apomictic potential in seven polyploid breeding lines of guayule, based on embryo production in flowers cultured in vitro ${ }^{\mathrm{z}}$ and orthogonal contrasts against a bulked diploid (sexual) control (in vitro Expt. 3).

\begin{tabular}{|c|c|c|c|c|}
\hline Line & $\begin{array}{c}\text { Flower } \\
\text { heads (no.) }\end{array}$ & $\begin{array}{l}\text { Flowers with } \\
\text { embryos } \\
(\% \pm \mathrm{SE})\end{array}$ & $\begin{array}{l}\text { Orthogonal contrasts } \\
\text { against diploid } \\
\text { controls }(P>\mathrm{F})\end{array}$ & $\begin{array}{l}\text { Apomictic } \\
\text { potential }\end{array}$ \\
\hline$\overline{\text { N9-5 }}$ & 15 & $22 \mathrm{a}^{\mathrm{y}}( \pm 7)$ & 0.027 & $\operatorname{High}^{\mathrm{x}}$ \\
\hline N7-11 & 29 & $21 \mathrm{a}( \pm 5)$ & 0.005 & High \\
\hline G7-11 & 14 & $20 \mathrm{ab}( \pm 9)$ & 0.013 & High \\
\hline P2-BK & 42 & $9 \mathrm{abc}( \pm 2)$ & 0.064 & Medium \\
\hline G7-15 & 29 & 9 abc $( \pm 4)$ & 0.150 & Medium \\
\hline P10-13 & 22 & $5 \mathrm{bc}( \pm 3)$ & 0.406 & Low \\
\hline G7-14 & 8 & $0 \mathrm{c}$ & 0.900 & None (sexual) \\
\hline Diploid & 15 & $0 \mathrm{c}$ & & None (sexual) \\
\hline
\end{tabular}

${ }^{\bar{z}}$ Flower heads were cultured in autoclaved Nitsch and Nitsch (1969) basal medium without growth regulators.

yMean separation within column by Duncan's multiple range test, $P \leq 0.05$.

${ }^{\mathrm{x}}$ Classifications were based as follows: High = mean embryo production significantly different from that of the diploid control, according to both Duncan's multiple range test and orthogonal contrast $(P \leq 0.05)$; Medium = mean embryo production not significantly different that of either the High group or the diploid control, according to Duncan's multiple range test; Low = mean embryo production significantly different from that of the High group, but not from that of the diploid control, according to Duncan's multiple range test; and None (sexual) = no embryo production. 
lack of medium effect for ovule and embryo culture of hybrids of another composite genus, Helianthus L. (sunflower).

Results with different media, and inclusion or exclusion of growth regulators, led us to the decision that in vitro floral culture in NN medium without growth regulators would be an adequate technique for estimation of AP in guayule. Using this technique, the seven lines used in this study fell into four AP classes, ranging from None (Sexual) to High (Table 6). We expected the diploid controls to be purely sexual, based on the reports of Gerstel (1950), Gerstel and Riner (1950), and Powers and Rollins (1945). This proved to be the case, because they never produced embryos in vitro.

Characterization of AP of the lines tested in this study were similar when based on results of either the in vivo or in vitro tests, but there were some important differences. Line P10-13 was ranked among the top three for embryo production in vivo, but decreased in the Low AP class in vitro. Line N9-5 was ranked together with G7-14 in vivo, but the AP classes of these lines in vitro were High and None, respectively. While the in vivo tests were simpler to perform than the in vitro technique, complications caused by pollen release inside the isolation bags and adverse environmental factors probably rendered it less accurate.

In all in vitro trials, even with $\mathrm{NAA}, \mathrm{GA}_{3}$, or kinetin in the medium, the embryos never developed sufficiently to permit macroscopic observation. Thus, it was necessary to use a simple ovule excision/squash technique and microscopic examination to score flowers. The embryos had ample time for development in vitro, because the length of the culture period was based on the fact that embryos resulting from controlled crosses reached mature size in 14 d (Esau, 1946; Keys and Ray, unpublished data). In contrast, in several members of the Poaceae, embryos developed after auxin treatment to the extent that apomictic seeds could be scored visually (Matzk 1991; Mazzucato et al. 1996). Helianthus annuus L. (common sunflower) also exhibited parthenogenesis in vitro (Yan et al., 1989). In that study, the highest rate of embryo formation also occurred without growth regulators in the medium and embryo development advanced only to the early heart-shaped stage, results that were similar to ours with guayule. For embryo rescue in hybrids of Helianthus, in which embryos are formed prior to culture, Espinasse et al. (1991) incorporated NAA into the nutrient medium to stimulate embryo elongation.

Our results with in vitro flower culture demonstrate that parthenogenesis in guayule is not dependent upon occurrence of pseudogamy, because pollination never occurred in the flowers. This separation of parthenogenesis and pseudogamy was also evident in grass species (Matzk, 1991; Mazzucato et al., 1996).

The effects of genotype $\times$ environment interactions on apomixis in guayule are unknown. Mamood et al. (1990) found seasonal differences in seed production that might be attributable to effects of high temperature stress on expression of apomictic potential. Seasonal differences in expression of apomixis in field experiments have been reported in other species, such as Pennisetum ciliare (L.) Link (buffelgrass) (Hussey et al., 1991), Paspalum cromyorrhizon Trin. (bahia grass) (Quarin, 1986), and Hyparrhenia hirta (L.) Stapf (coolatai grass) (McWilliam et al., 1970). Under controlled conditions, reproductive mode was influenced by photoperiod in $P$. cromyorrhizon (Quarin, 1986), and Themeda australis (R. Br.) Stapf (kangaroo grass) (Evans and Knox, 1969). In our trials, guayule plants grown in the greenhouse required a 14-h photoperiod to stimulate flowering, suggesting there is a photoperiod effect for this species. The in vitro technique described herein would permit the study of environmental effects on floral development and expression of apomixis in guayule.
The presence of plants exhibiting parthenogenesis within lines classed as having low AP, and sexual plants within lines classed as having high AP, means that a pool of plant material could be generated to permit the study of the molecular control of reproductive mode in guayule. With more isogenic plant material, differential screening of cDNA libraries (Smith, 1993; Matzk et al., 1995) or differential display (Liang and Pardee, 1992; Vielle-Calzada et al., 1996) could reveal control mechanism(s). This information, in conjunction with results of in vitro tests to determine effects of environmental factors, would contribute significantly to understanding the molecular genetics controlling the mode of reproduction in this species.

\section{Literature Cited}

Artschwager, E. 1943. Contribution to the morphology and anatomy of guayule (Parthenium argentatum). USDA Bul. 342

Esau, K. 1946. Morphology of reproduction in guayule and certain other species of Parthenium. Hilgardia 7:61-120.

Espinasse, A., J. Volin, C.D. Dybing, and C. Lay. 1991. Embryo rescue through in ovulo culture in Helianthus. Crop Sci. 31:102-108.

Evans, L.T. and R.B. Knox. 1969. Environmental control of reproduction in Themeda australis. Austral. J. Bot. 17:375-389.

Gerstel, D.U. 1950. Self-incompatibility studies in guayule II. Inheritance. Genetics 35:482-506.

Gerstel, D.U. and M.E. Riner. 1950. Self-incompatibility studies in guayule I. Pollen tube behavior. J. Hered. 41:49-55.

Hussey, M.A., E.C. Bashaw, K.W. Hignight, and M.L. Dahmer. 1991. Influence of photoperiod on the frequency of sexual embryo sacs in facultative apomictic buffelgrass. Euphytica 54:141-145.

Liang, P. and A.B. Pardee. 1992. Differential display of eukaryotic messenger RNA by means of the polymerase chain reaction. Science 257:967-971.

Lloyd, G.B. and B.H. McCown. 1980. Commercially feasible micropropagation of mountain laurel, Kalmia latifolia, by use of shoot-tip culture. Proc. Intl. Plant Prop. Soc. 30:421-437.

Mamood, A.N., D.T. Ray, and G.D. Waller. 1990. Seed set in guayule (Parthenium argentatum, Asteraceae) in relation to insect pollination. Econ. Bot. 44:440-444.

Matzk, F. 1991. A novel approach to differentiated embryos in the absence of endosperm. Sex. Plant Reprod. 4:88-94.

Matzk, F., H.M. Meyer, H. Bäumlein, and H.J. Balzer. 1995. A novel approach to the analysis of the initiation of embryo development in Gramineae. Sex. Plant Reprod. 8:266-272.

Mazzucato A., A.P.M. den Nijs, and M. Falcinelli. 1996. Estimation of parthenogenesis frequency in Kentucky bluegrass with auxin-induced parthenogenetic seeds. Crop Sci. 36:9-16.

McWilliam, J.R., K. Shanker, and R.B. Knox. 1970. Effects of temperature and photoperiod on growth and reproductive development in Hyparrhenia hirta. Austral. J. Agr. Res. 21:557-569.

Nitsch, J.P. and C. Nitsch. 1969. Haploid plants from pollen grains. Science 163:8587.

Powers, L. and R.C. Rollins. 1945. Reproduction and pollination studies in Parthenium argentatum Gray and P. incanum H.B.K. J. Amer. Soc. Agron. 37:184-193.

Quarin, C.L. 1986. Seasonal changes in the incidence of apomixis of diploid, triploid, and tetraploid plants of Paspalum cromyorrhizon. Euphytica 35:515522.

Ray, D.T., D.A. Dierig, A.E. Thompson, and T.A. Coffelt. 1999. Registration of six germplasm lines of guayule with high yielding ability. Crop Sci. 39:300.

SAS Inst., Inc. 1988. SAS/STAT user's guide: Release 6.03 ed. SAS Inst., Inc., Cary, N.C.

Smith, A.G. 1993. Isolation and regulation of differentially expressed genes from flowers, p. 117-139. In: B.R. Jordan (ed.). The molecular biology of flowering. CAB Intl., Oxon, United Kingdom.

Thompson, A.E. and D.T. Ray. 1988. Breeding guayule. Plant Breeding Rev. 6:93165.

Vielle-Calzada, J.-P., M.L. Nuccio, M.A. Budiman, T.L. Thomas, B.L. Burson, M.A. Hussey, and R.A. Wing. 1996. Comparative gene expression in sexual and apomictic ovaries of Pennisetum ciliare (L.) Link. Plant Mol. Biol. 32:10851092.

Yan, H., H.-Y. Yang, and W.A. Jensen. 1989. An electron microscope study on in vitro parthenogenesis in sunflower. Sex. Plant Reprod. 2:154-166. 